\title{
LSB Steganography in Color and Grayscale Images without using the Transformation
}

\author{
G. Viji and J. Balamurugan
}

\begin{abstract}
A technique which enables to have a secret communication in modern technology using public channel is known as steganography This paper deals with constructing and implementing new algorithm based on hiding a large amount of payloads (image, audio, text) file into color image. We have been used adaptive image filtering and adaptive image segmentation with bits replacement on the appropriate pixels. The information has to be hidden in the LSB of the cover image, so there is no much chances present in the embedded image. High security layers have been proposed through three layers to make it difficult to break through the encryption of the input data and confuse steganalysis too. We show that our algorithm can embed efficiently a large amount of data that has been reached to $90 \%$ of the image size with high quality of the output. . It is observed that the proposed algorithm has excellent PSNR, provides high level security and more robust compared to individual transform techniques.
\end{abstract}

Keywords--- LSB, PSNR, Security, Steganography

\section{INTRODUCTION}

$S^{T}$ TEGANOGRAPHY is derived from the Greek word steganos which literally means "covered" and graphia which means "writing", i.e. covered writing [1], [8]. Currently, research in steganography has grown explosively in terms of further exploring message hiding within an object, a text or even a picture. Steganography often draws confusion with cryptography [2] in terms of appearance and usage. The most significant difference between steganography and cryptography is the suspicion factor. In fact, when both cryptography and steganography are being implemented together, an acceptable amount of security could be achieved. Hence, a security utility which is built to essentially protect the privacy of confidential data transmission of information is needed. This kind of method is used to make the presence of a secret data appear invisible to eavesdroppers such as keyloggers or harmful tracking cookies which can monitor a user's keystroke when entering password and personal information. Information theft by malware has been widely used [4] to capture user's password and confidential data in order to use it for hijacking personal possession especially counterfeiting or falsification of credit cards and identification cards. Therefore, the proposed method can overcome this problem by embedding the data into images so that it can be sent to the other party as an innocent looking file through the internet or a public domain during information exchange as in [8].

G. Viji, Lecturer, M.Kumarasamy college of Engineering, Karur-113

J. Balamurugan, PG student, M.Kumarasamy College of Engineering, Karur-113. E-mail: balamuruganec711@ gmail.com
Therefore, it is essential to prevent the interception by other parties while transmitting data for safeguarding human to human communications. Basically, the secret data refers to a message which is saved as a text file that needs to be hidden. In this application, a GIF image will be chosen as a cover medium. The stego-image is the final product after a secret message is embedded in the cover object. A secret message will be concealed in a cover-image by applying an embedding algorithm to produce a stego-image. The transmission of the stego-image via a communication channel is performed by a sender to a receiver. To reveal the covert message that is concealed by the sender, the receiver needs to have the destego algorithm which is parameterized by a stego-key to extract the secret message. This is the purpose of a steganographic system where an attacker who does not possess the name of a file or the stego-key for accessing it definitely will not be able to determine whether the file is even present [7]. In an efficient steganographic system, a normal cover medium should not be distinguishable from a stegoobject [6]. Steganography Mechanism Digital images have become commonplace and nowhere are these images more prevalent than on the World Wide Web in the Internet [8]. Using digital images as a carrier medium is suitable for information hiding because of their insensitivity for the human visual system [11]. The vast majority of web pages are impressively sophisticated with colour images and thus Internet users browsing through the web no longer pay attention to sites containing images or to the downloading of images and data files from the Web [8]. Besides, there is a large amount of redundant bits in an image. The redundant bits of an object are those bits that can be altered but the alteration cannot be visibly detected by human eyes [3].

\section{LITERATURE REVIEW}

This section reviews on the least significant bit (LSB) insertion method and the significance of using steganography for color image.

\section{A. Least Significant Bit Insertion Method}

Least significant bit insertion is a common, simple approach to embed information in a cover file [6], [7]. The LSB is the lowest order bit in a binary value. This is an important concept in computer data storage and programming that applies to the order in which data are organized, stored or transmitted [1]. Usually, three bits from each pixel can be stored to hide an image in the LSBs of each byte of a 24-bit image. Consequently, LSB requires that only half of the bits in an image be changed [7] when data can be hidden in least and second least significant bits and yet the resulting stego-image which will be displayed is indistinguishable to the cover image to the human visual system [7]. 
In one byte, the 1 bit LSB is indicated:

$$
\begin{array}{|l|l|l|l|l|l|l|l|}
\hline 1 & 0 & 0 & 1 & 0 & 0 & 0 & 1 \\
\hline
\end{array}
$$

Figure 1: Least Significant Bit

The last bit of the byte is selected as the least significant bit (as illustrated in Figure 1) because of the impact of the bit to the minimum degradation of images [1]. The last bit is also known as right-most bit, due to the convention in positional notation of writing less significant digit further to the right [7].

In bit addition (refer to Figure 3), the least significant bit has the useful property of changing rapidly if the number changes slightly. For example, if 1 (binary 00000001) is added to 3 (binary 00000011), the result will be 4 (binary 00000100) and three of the least significant bits will change (011 to 100). The one bit addition is done as shown in Fig.2

1 Bit Addition 1+2=3)

i) Value inserted

ii)

\begin{tabular}{|l|l|l|l|l|l|l|l|}
\hline 0 & 0 & 0 & 0 & 0 & 0 & 0 & 1 \\
\hline
\end{tabular}

Original Value

\begin{tabular}{|l|l|l|l|l|l|l|l|}
\hline 0 & 0 & 0 & 0 & 0 & 0 & 1 & 1 \\
\hline
\end{tabular}

iii)

Final modified Value

\begin{tabular}{|l|l|l|l|l|l|l|l|}
\hline 0 & 0 & 0 & 0 & 0 & 1 & 0 & 0 \\
\hline
\end{tabular}

Figure 2: Example of bit addition

Basically, by modifying the insignificant bits, the cover image is typically altered in a nearly imperceptible manner thereby ensuring that any observer would be unaware of the alteration made. Employing the LSB technique for data hiding achieves both invisibility and reasonably high storage payload, a maximum of one bit per pixel (bpp) for grayscale and three bpp for Red-Green- Blue (RGB) images [3]. There are a number of steganographic tools which employ LSB insertion methods available on the web.

\section{PROPOSED METHOD}

The approach proposed is simple for implementation when compared to Pixel value Differencing (PVD) method and yet achieves a High security and imperceptibility. This paper presents a "Steganography for color image based on adaptive image segmentation".

The basic flow of the proposed method is shown in the Fig.3. In this section we describe our proposed method. The color image is used as a cover image. The proposed steganography for color image can be done by performing the following steps:

\section{A. The First Part Is Used To Hide Data File Inside Bitmap According To The Following Action}

- Accept encryption password from the sender.

- Find a maximum size (number of bytes) that is accepted by the cover-image.
- Perform compression on secret data file to increase the amount of hiding secret data.

- Perform encryption on secret data file.

- Adaptive segmentation according to the Password

- Perform scanning to select suitable pixels on each segment by extracting image characteristics. The candidate pixels are used to embed secret data.

- Perform hiding of the secret data into bitmap images according to color characteristics.

B. While the second part is used to extract data from bitmap image at the receiver side in conformity with the

following actions:

- Extracting password.

- Scanning segment's pixels according to the password.

- Extracting data file.

- Decrypt the extracting data file by using same password which is used for the encryption

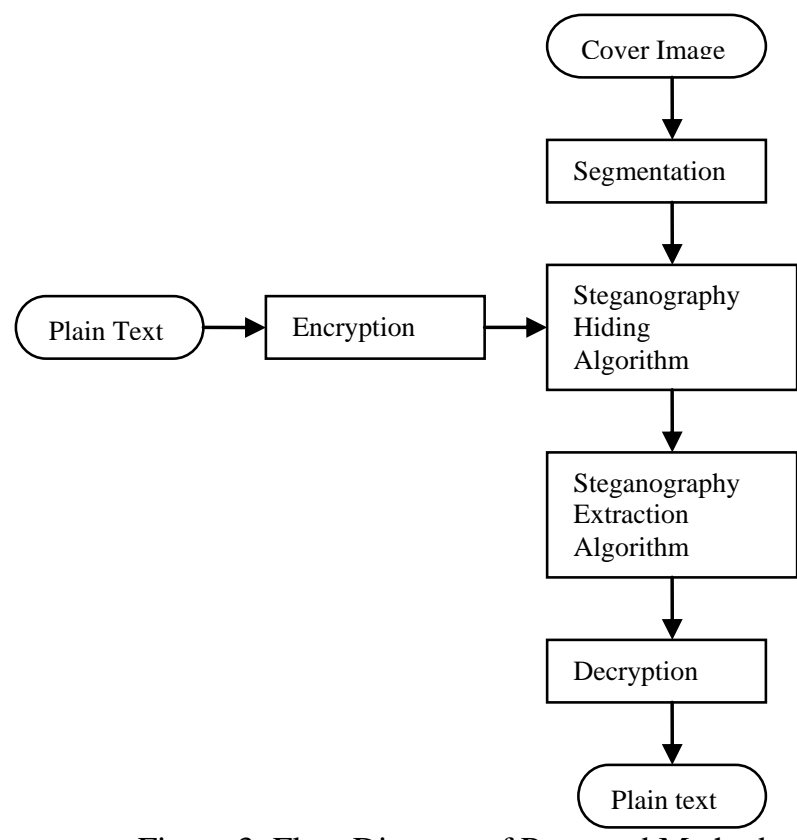

Figure 3: Flow Diagram of Proposed Method

\section{Segmentation}

The cover image which is used here is a color image so, first the three primary colors(R, G, B) are separated. Then the each color is then split into six sub blocks this method is known as blocking. The data hiding is done manually so that in which block the data has to be hidden.

If the image size, say $1000 \times 750$ pixels (written as width $\mathrm{x}$ height by convention), then there would be 1000 columns and 750 rows of data values, or $1000 \times 750=750000$ pixels total. For 24 bit color, each pixels data contains three 8-bit RGB byte values, or $750,000 \times 3=2,250,000$ bytes. Every pixel is the same size, because a pixel is simply the color of the area between the grid lines. The area will be colored by the one corresponding RGB data value. Larger areas of the same color are just many multiple identical pixels, including the blank 
background (for example the blue sky below), which are many more pixels too.

The 24 bit RGB color images use 3 bytes, and can have 256 shades of red, 256 shades of green and 256 shades of blue. Therefore $256 \times 256 \times 256=16.7$ million possible combinations or colors for 24 bit RGB color images. The composite of the RGB values creates the final color for one pixel area.

\section{Encryption}

Encryption is the process of transforming information (referred to as teximage) using an algorithm to make it unreadable to anyone except those possessing special knowledge, usually referred to as a key. The result of the process is encrypted information.

\section{E. Decryption}

Decryption is the reverse process of the encryption. For that the same algorithm is used. Then the output of it will the plain text and the image the same technique is to be followed to retrieve the image and the textimage.

Table 1: Decryption Time Using Brute Force Attack Method

\begin{tabular}{|c|c|}
\hline $\begin{array}{c}\text { Key Length } \\
\text { (in bits) }\end{array}$ & Time to Decrypt \\
\hline 10 & Less than 1 second \\
\hline 20 & 21 seconds \\
\hline 30 & 6 hours \\
\hline 40 & 255 days \\
\hline 64 & Almost 12,000 years \\
\hline 128 & $\begin{array}{l}\text { Over } 200 \text { septillion years(a number } \\
\text { with } 27 \text { digits), longer than life of the } \\
\text { universe }\end{array}$ \\
\hline
\end{tabular}

\section{RESUltS AND DisCUSSION}

\section{A. Obtaining Secret Message}

In the previous methods, only a single text file can be hidden in and image. This is a major drawback while hiding large amount of data. This can be overcomed by using this recent technique in which six text files can be hidden in a single image simultaneously.

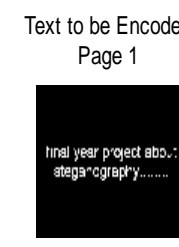

Page 4

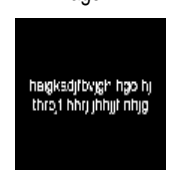

Page 2

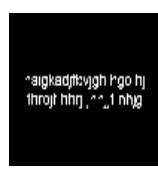

Page 5

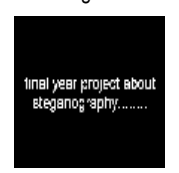

Page 3

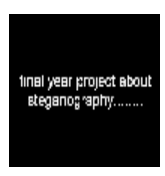

Page 6

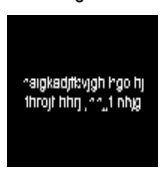

Figure 4: Secret Message

\section{B. First Level Encrypted Image}

The cover image obtained in which data is to be hidden in the form of text files. The processed teximage is to be hidden in the RGB components of the cover image. Thus, in the next stage, the individual R,G and B components are separated and labeled individually.

In this part the desire text images (max 6 images) are going to encoded with the Color image. At first pair of text images are combined into a single image. Simple logic is used to achieve this. Pixels are selected from alternate location of pair of text images. As a result of this, image having details (but alternate locations) about pair of two text images. Further explained that if a location $(n, m)$ have the pixel of Image 1 next location $(n+1, m+1)$ have the pixel taken from Image 2 . This task is done by alterim() function. Two arguments of this function are two text images. Next step is to encode the text image which was already obtained from the result of alterim() function. We obtained 3 single text images from this function. Each image is encoded in each color frames R,G and B respectively

\section{First Level Encripted Text Page 1}

Page 2

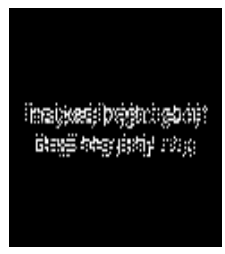

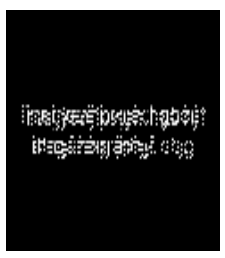

Page 3

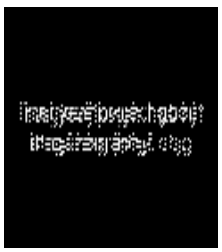

Figure 5: Encrypted Image

\section{Comparison of the Cover Image before and After Embedding the Secret Data}

The processed teximage is to be hidden in the RGB components of the cover image separately. i.e teximage 1 is hidden in $\mathrm{R}$ component, teximage 2 is hidden in $\mathrm{G}$ component and teximage 3 is hidden in $\mathrm{B}$ component of the cover image. This process is done by LSB substitution algorithm. By using this process, image degradation is very much reduced which is almost negligible.

\section{Color image (Before Encoding)}

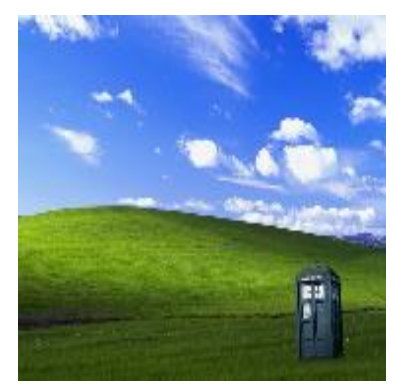

Stegano image (After Encoding)

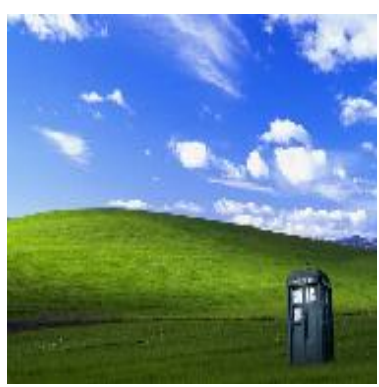

Figure 6: Comparison of the Input \& Output Image

By using LSB algorithm, the least significant bit is the only altered bit of information and hence there is no drastic or notable change or variation in the cover image 


\section{Inappropriated Decrypted Image}

If they give password as wrong in more than three times the image is destroyed.

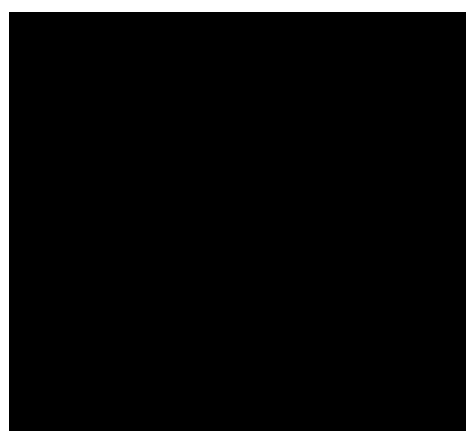

Figure 7: Inappropriated Image

\section{E. Accuracy Calculation}

By comparing the output image i.e., embedded cover image with the our original image pixel values, we calaculate the accuracy of the proposed method.For this method we can obtain maximum of $96.8 \%$ of accuracy.

Mean Square Error (MSE): It is the measure used to quantify the difference between the initial and the distorted or noisy image. Let Pi represents the pixel of one image of size $\mathrm{N}$ and Qi that of the other.

$$
M S E=\sum_{i=1}^{\text {allpixelisallpixels }} \sum_{i=1}^{(\text {Cover }(i, j)-\text { stegd }(i, j))^{2}}
$$

From MSE we can find Peak Signal to Noise Ratio (PSNR) to access the quality of the Stego image with respect to cover image given by

$$
P S N R=20 \log _{10} \frac{255}{\sqrt{M S E}}
$$

\section{CONCLUSION}

Thus from the method which is proposed in this paper, the number of bits to be hide is very high because more number of bits are available in color image. The security of the hidden message is very high by using the password when compared to the other methods. The image quality is also better.The Entropy, MSE and Capacity are improved with acceptable PSNR compared to the existing algorithm. In future the algorithm can be tested with the transform techniques.

\section{REFERENCES}

[1] Weiqi Luo, , Fangjun Huang, and Jiwu Huang, "Edge Adaptive Image Steganography Based on LSB Matching Revisited", IEEE Transactions Forensics and Security, Vol 5 ,No. 2, June 2010.

[2] Alain Brainos, "A Study of steganography and the Art of Hiding Information", SecurityWriter, July 27, (2004).

[3] Chandramouli R and Memon N, "Analysis of LSB based image steganography techniques", Proceedings 2001 International Conference on Image, Vol. 3, pp. 1019-1022.

[4] Der-Chyuan Lou and Jiang-Lung Liu, "Steganographic Method for Secure Communications", Elsevier Science Ltd, Vol 21, No 5, 2002, pp 449-460.

[5] Mohammed Al-Mualla and Hussain Al- Ahmad, "Information Hiding: steganography and Watermarking". Proceedings of the IEEE ,(2008).

[6] Fabien A. P. Petitcolas, Ross J. Anderson and Markus G. Kuhn. "Information Hiding - A Survey", Proceedings of the IEEE, special issue on protection of multimedia content, pp. 1062-1078, July (1999).

[7] Morkel.T, J.H.P. Eloff, M.S. Olivier, "An Overview of Image Steganography", Proceedings of the Fifth Annual Information Security South Africa Conference, Sandton, South Africa, (2005).

[8] Neil F., Zoran D., and Sushil J., "Information Hiding Steganography and Watermarking Attacks and Countermeasures", Printed in United State of America, (2000).

[9] Pierre Moulin, and Joseph A, " Information Theoretic Analysis of Information Hiding," O'Sullivan University of Illinois Washington University Beckman Inst., October (1999).

[10] Roshidi Din and Hanizan Shaker Hussain, "The Capability of Image In Hiding A Secret Message", Proceedings of the 6th WSEAS International Conference on Signal, Speech and Image Processing, September (2006).

[11] Y. Q. Shi et al., "Image steganalysis based on moments of characteristic functions using wavelet decomposition, prediction-error image, and neural network," in Proc. IEEE Int. Conf. Multimedia and Expo, 2005.

[12] B. Li, J. Huang, and Y. Q. Shi, "Textural features based universal steganalysis," Proc. SPIE on Security, Forensics, Steganography and Watermarking of Multimedia, vol. 6819, 2008.

[13] M. Goljan, J. Fridrich, and T. Holotyak, "Newblind steganalysis and its implications," Proc. SPIE on Security, Forensics, Steganography and Watermarking of Multimedia, vol. 6072, pp. 1-13, 2006.

[14] K. Hempstalk, "Hiding behind corners: Using edges in images for better steganography," in Proc. Computing Women's Congress, Hamilton, New Zealand, 2006.

[15] K. M. Singh, L. S. Singh, A. B. Singh, and K. S. Devi, "Hiding secret message in edges of the image," in Proc. Int. Conf. Information and Communication Technology, pp. 238-241,2007.

[16] M. D. Swanson, B. Zhu, and A. H. Tewfik, "Robust data hiding for images," in Proc. IEEE on Digital Signal Processing Workshop, Sep.1996.

[17] D. Wu and W. Tsai, "A steganographic method for images by pixelvalue differencing," Pattern Recognit. Lett., vol. 24, pp. 1613-1626,2003.

[18] X. Zhang and S. Wang, "Vulnerability of pixel-value differencing steganography to histogram analysis and modification for enhanced security," Pattern Recognit. Lett., vol. 25, pp. 331-339, 2004.

[19] C. H. Yang, C. Y. Weng, S. J. Wang, and H. M. Sun, "Adaptive data hiding in edge areas of images with spatial LSB domain systems," IEEE Trans. Inf. Forensics Security, vol. 3, no. 3, pp. 488-497, 2008.

[20] M. Kharrazi, H. T. Sencar, and N. Memon, "Cover selection for steganographic embedding," in Proc. IEEE Int. Conf. Image Processing, pp. 117-120, 2006.

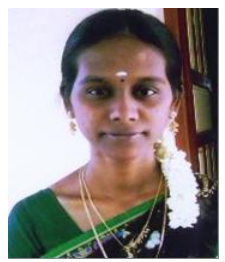

G. Viji has finished M.E(communication systems) in Mepco schlenk Engineering College, Sivakasi,. She has finished B.E(ECE) in PSR engineering college ,sivakasi.Now she is working in lecturer as M.Kumarasamy college of engineering,karur.Her area interest like digital image processing,communication system

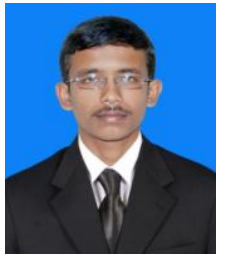

J. Balamuurgan has finished B.E(ECE) in M.Kumarasamy college of engineering, Karur .Now he is working in CTO as M.Kumarasamy college of engineering,karur.His area interest like digital image processing, Microprocessor 